https://doi.org/10.15407/ujpe64.12.1118

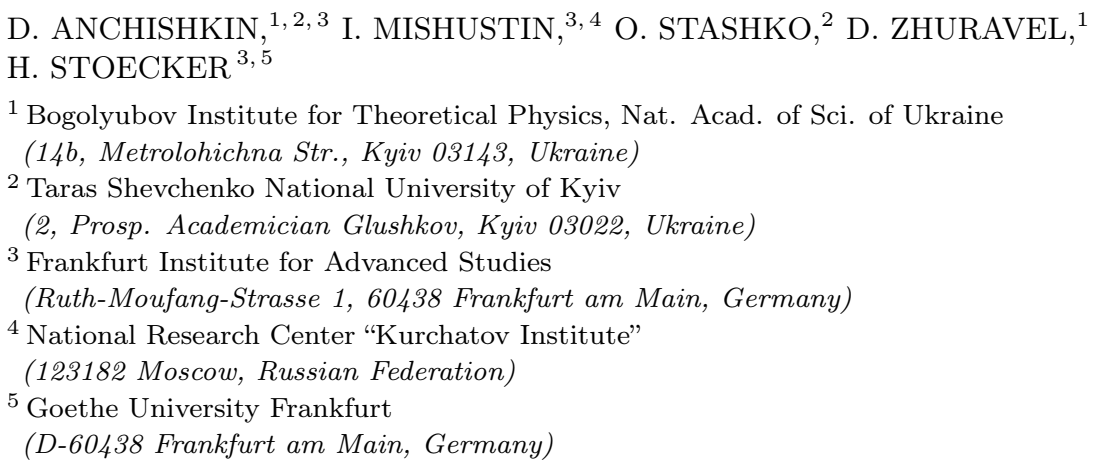

\title{
FINITE-TEMPERATURE BOSE-EINSTEIN CONDENSATION IN INTERACTING BOSON SYSTEM ${ }^{1}$
}

\begin{abstract}
Thermodynamical properties of an interacting boson system at finite temperatures and zero chemical potential are studied within the framework of the Skyrme-like mean-field toy model. It is assumed that the mean field contains both attractive and repulsive terms. Self-consistency relations between the mean field and thermodynamic functions are derived. It is shown that, for sufficiently strong attractive interactions, this system develops a first-order phase transition via the formation of a Bose condensate. An interesting prediction of the model is that the condensed phase is characterized by a constant total density of particles. It is shown that the energy density exhibits a jump at the critical temperature.

Ke ywords: pion gas, phase transition, condensate.
\end{abstract}

\section{Introduction}

In recent years, the properties of a hot and dense hadronic matter have attracted considerable interest. Such matter can be produced in relativistic nucleus-nucleus collisions which are under investigations in many laboratories. QCD-motivated effective models and lattice simulations indicate that the chiral symmetry restoration and the deconfinement phase transition should take place at high temperatures and particle densities. In this paper, we study specifically the properties of interacting boson systems in the framework of a toy model using a thermodynamically consistent mean-field approach. The model aims to estimate the scale and relation of attractive and repulsive contributions to the potential in order to investigate a possibility of the Bose-Einstein condensation of interacting bosonic particles. This problem has been studied previously, starting from the pioneer works of A.B. Migdal and coworkers [1-4] and later by many authors using different models and meth-

(C) D. ANCHISHKIN, I. MISHUSTIN, O. STASHKO,

D. ZHURAVEL, H. STOECKER, 2019 1118 ods. A possible formation of classical pion fields in relativistic nucleus-nucleus collisions was discussed in Refs. [5-8]. In more recent studies [9-13], pionic systems with a finite isospin chemical potential at low temperatures have been considered. Interesting new results concerning dense pionic systems have been obtained recently using lattice methods $[14,15]$.

In the present paper, we consider interacting boson systems at zero chemical potential, but high temperatures, where thermally produced particles have rather high densities. Simple calculations for noninteracting hadron resonance gas show that the particle density may reach values $(0.1-0.2) \mathrm{fm}^{-3}$ at temperatures $100-150 \mathrm{MeV}$, which are below the deconfinement phase transition, see, e.g., Refs. [16, 17]. Under such conditions, the interaction effects should become important. To account for the interaction between the bosons, we introduce a phenomenological Skyrme-like mean field $U(n)$, which depends on the

1 This work is based on the results presented at the XI BolyaiGauss-Lobachevskii (BGL-2019) Conference: Non-Euclidean, Noncommutative Geometry and Quantum Physics.

ISSN 2071-0194. Ukr. J. Phys. 2019. Vol. 64, No. 12 
particle density $n$ only. Then the thermodynamical consistency relations are used to calculate the particle density, energy density, and pressure as functions of the temperature. An important distinctive feature of the considered system is that, in contrast to, for example, the bosonic matter, the number of bosons is not conserved, but is determined by the minimization of a thermodynamic potential.

\section{Interacting Boson Systems with Zero Chemical Potential}

\subsection{Pressure, energy density, and particle-number density in the gas phase}

Let us consider firstly the interacting bosonic system without condensate. We call this state a liquid-gas phase in order to distinguish it from a weakly interacting pion gas. As an instrument for our investigation, we use the "Thermodynamic mean-field model", see Ref. [18] (for early works, see [19, 20]). In this approach, the influence of the medium on a particle is realized as the mean-field $U(n)$, which shifts the free dispersion relation so that the single-particle energy reads

$E(\boldsymbol{k}, n)=\sqrt{m^{2}+\boldsymbol{k}^{2}}+U(n)$,

where $n$ is the particle-number density, and $T$ is the temperature. The presence of the fields in the system, which are responsible for interactions between particles, results not just in a distortion of the free single-particle dispersion relation like in (1), but also gives its own contribution to the energy density and produces an excess pressure $P_{\text {ex }}(n)$. Then the pressure of the homogeneous system of interacting bosons with $\mu=0$ in the grand canonical ensemble looks like

$p(T)=\frac{g}{3} \int \frac{d^{3} k}{(2 \pi)^{3}} \frac{\boldsymbol{k}^{2}}{\sqrt{m^{2}+\boldsymbol{k}^{2}}} f(\boldsymbol{k} ; T)+P_{\mathrm{ex}}(n)$,

where the distribution function reads

$f(\boldsymbol{k} ; T)=\left\{\exp \left[\frac{E(\boldsymbol{k}, n)}{T}\right]-1\right\}^{-1}$.

The energy density in the liquid-gas phase is obtained from the Euler relation $\varepsilon+p=T s+\mu n$, where the entropy density is the derivative $s=\partial p(T) / \partial T$. Hence, the energy density reads

$\varepsilon(T)=g \int \frac{d^{3} k}{(2 \pi)^{3}} \sqrt{m^{2}+\boldsymbol{k}^{2}} f(\boldsymbol{k} ; T)+\varepsilon_{\mathrm{ex}}(n)$,

ISSN 2071-0194. Ukr. J. Phys. 2019. Vol. 64, No. 12 with

$\varepsilon_{\mathrm{ex}}(n) \equiv n U(n)-P_{\mathrm{ex}}(n)$.

In fact, the quantities $U(n, T)$ and $P_{\mathrm{ex}}(n, T)$ are due to some interaction between particles in the many-particle system, i.e., because the source of these two quantities is the same, they are related to one another as (see [18])

$n \frac{\partial U(n, T)}{\partial n}=\frac{\partial P_{\mathrm{ex}}(n, T)}{\partial n}$

To resolve the model, it is necessary, first, to solve the self-consistent equation for the particle density $n$ for every given temperature $T$

$n=\frac{g}{2 \pi^{2}} \int_{0}^{\infty} d k k^{2}\left\{\exp \left[\frac{E(\boldsymbol{k}, n)}{T}\right]-1\right\}^{-1}$

In this paper, we consider pion systems with $\mu=0$. Then a nonzero pion density is only possible at $T>0$.

\section{Onset of the Bose Condensation}

The Bose-Einstein distribution function (3) implies that, at the momentum $\boldsymbol{k}=0$, the single-particle energy should satisfy the condition $E(\mathbf{0}, n) \geq 0$. Otherwise, the distribution function (3) takes negative values. Exactly this condition determines the interval of temperatures, where the Bose condensation occurs.

The limiting density of thermal Bose particles, $n_{\text {lim }}(T)$, just before the formation of a Bose condensate, i.e. at $U(n)=-m$, is the same as in the ideal gas at $\mu=m$ :

$n_{\lim }(T)=\frac{g}{2 \pi^{2}} \int_{0}^{\infty} d k k^{2}\left[\exp \left(\frac{\sqrt{m^{2}+k^{2}}-m}{T}\right)-1\right]^{-1}$.

In Fig. 1 (lower panel), this dependence is depicted as a red-dashed line, which separates the normal phase (which contains thermal particles) from the phase with a Bose condensate. Further, we come to the general conclusion: for mean-field potentials deeper than $-m$, the equilibrated bosonic system will develop a Bose condensate.

\subsection{Skyrme-like parametrization of the mean field}

The formalism described in the previous section has been applied to several physically interesting systems

1119 



Fig. 1. Top panel: Mean field $U(n)$ versus the particle density for different values of the parameter $\kappa=A / A_{\mathrm{c}}$. The densities $n_{1}, n_{2}$ and $n_{0}$ are the crossing points with the line $U=-m_{\pi}$. Lower panel: particle density versus the temperature for the interacting pion system with $\mu=0$. The temperatures $T_{1}, T_{2}$, and $T_{0}$ indicate the points, where the densities $n_{1}, n_{2}$, and $n_{0}$ cross the limiting density line $n_{\lim }(T)$ (red dashed curve)

including the hadron-resonance gas [18] and the pionic gas [21]. In the present study, we extend this approach to the case of a bosonic system which potentially can undergo the Bose condensation. To illustrate this possibility, we formulate the Skyrme-like toy model [22], where we assume that the interaction between particles is described by the mean field

$U(n)=-A n+B n^{2}$,

where $n$ is the particle density, $A>0$ and $B>0$ are the positive model parameters, which should be specified for each particle species. Hence, in our calculations, we consider a general case to study a bosonic system with both attractive and repulsive contributions to the mean field (8). Some additional contribution to the attractive mean field at high energies or high temperatures, $(T \propto 150 \mathrm{MeV})$, may be provided by other hadrons present in the system.

The excess pressure is obtained using the condition of thermodynamic consistency (5) with $U(n)$ from Eq. (8),

$P_{\mathrm{ex}}(n)=\int_{0}^{n} d n^{\prime} n^{\prime} \frac{\partial U\left(n^{\prime}\right)}{\partial n^{\prime}}=-\frac{1}{2} A n^{2}+\frac{2}{3} B n^{3}$,

where $\lim _{n \rightarrow 0} U(n)=0$ has been used.

To be specific in numerical calculations, we take bosons with the mass $m_{\pi}=139 \mathrm{MeV}$ and the degeneracy factor $g=3$, which we call "pions". For the repulsive coefficient $B>0$, we use a fixed value obtained from an estimate based on the virial expansion [23], $B=10 m_{\pi} b^{2}$ with $b$ equal to four times the proper volume of a particle, i.e. $b=16 \pi r_{0}^{3} / 3$. Below, we take $b=0.45 \mathrm{fm}^{3}$ that corresponds to a pion radius $r_{0} \approx 0.3 \mathrm{fm}$. The "repulsive" coefficient $B$ is fixed along the whole investigation in the present paper. The "attractive" coefficient $A$ is considered as a model parameter which will be varied in proper limits.

The potential $U(n)$ is shown in Fig. 1 (top panel) for several values of the parameter $\kappa=A / A_{\mathrm{c}}$, characterizing the strength of the attractive interaction. Here, $A_{\mathrm{c}}=2 \sqrt{m B}$ is the critical value of $A$ at which the minimum of the potential reaches the energy level $-m$. Below, we choose $\kappa$ as a variational parameter, i.e., we parametrize variations of the "attractive" coefficient $A$ by the dimensionless parameter $\kappa$. The values of $\kappa \geq \kappa_{\mathrm{c}}=1$ lead to the crossing of the $-m$ level and to the appearance of the density interval, where the function $E(\mathbf{0}, n)$ is negative. The end points of this interval are determined from the equation

$U(n)+m=0$.

With $U(n)$ from (8), the solutions of this equation are

$$
\begin{aligned}
& n_{1}=\sqrt{\frac{m}{B}}\left(\kappa-\sqrt{\kappa^{2}-1}\right), \\
& n_{2}=\sqrt{\frac{m}{B}}\left(\kappa+\sqrt{\kappa^{2}-1}\right) .
\end{aligned}
$$

In the interval $n_{1}<n<n_{2}$, the integral in Eq. (6) is not positive definite. Therefore, such densities cannot

ISSN 2071-0194. Ukr. J. Phys. 2019. Vol. 64, No. 12 
be realized in an equilibrium system. At $\kappa>\kappa_{\mathrm{c}}=1$, the change of the pion density from $n=n_{1}$ to $n=n_{2}$ is only possible via the condensation of pions in the zero-momentum mode, $|\boldsymbol{k}|=0$, so that their total density jumps from $n=n_{1}$ to $n=n_{2}$. As is seen from Eq. (11), the critical value of the parameter $A$ is obtained, when both roots coincide, i.e., when $\kappa=1$ or $A=A_{\mathrm{c}}=2 \sqrt{m B}$. For the parameter $B$ specified above, $A_{\mathrm{c}}=2 \sqrt{10} m_{\pi} b \approx 396 \mathrm{MeV} \cdot \mathrm{fm}^{3}$. The corresponding critical pion density, when the minimum of the potential reaches the level $-m_{\pi}$, is $n_{0}=A_{c} / 2 B=\frac{1}{\sqrt{10} b} \approx 0.7 \mathrm{fm}^{-3}$.

\subsection{Energy density and pressure in the gas-liquid phase}

The liquid-gas phase is distinguished by the values of the parameter $\kappa$ as $\kappa \leq 1$. Hence, it is assumed that $U(n) \geq-m$ for all $n$. To obtain the particle density $n(T)$, one has to solve the self-consistent equation (6) for the fixed value $\kappa \leq 1$ and for the given temperatures from some interval. On the lower panel in Fig. 1, we depicted the results of calculations of the pion density for several values of the parameter $\kappa=0,0.55,0.82,1$ as black solid lines. The dashed black curve represents the ideal pion gas with $\mu=0$.

Now, with the dependence $n(T)$ in hands, we can calculate the pressure and energy density in the gasliquid phase using Eqs. (2) and (4) with account for $P_{\mathrm{ex}}(n)$ from Eq. (9).

\subsection{Self-consistent solution for the mixed phase}

Let us return to Eq. (6), which determines the particle density as a function of the temperature. The solutions $n(T)$ have been found iteratively for several values of $\kappa$, as shown in Fig. 1. The limiting density $n_{\lim }(T)$, Eq. (7), is also shown in this figure. In a standard treatment of the Bose-Einstein condensation (see, e.g., [24]), the particle density above $T_{\mathrm{c}}$ consists of two contributions: "thermal" particles and "condensate" particles. Hence, in the mixed phase, the self-consistent equation (6) is generalized to (see $[22,24])$

$$
n=n_{\text {cond }}+\frac{g}{2 \pi^{2}} \int_{k \neq 0} d k k^{2}\left\{\exp \left[\frac{E(\boldsymbol{k}, n)}{T}\right]-1\right\}^{-1}
$$

where $n$ is the total particle-number density which consists from the density of thermal particles $n_{\text {th }}$ and the density of condensed particles $n_{\text {cond }}$, which have zero momentum. Note that, in our treatment, $T_{\mathrm{c}}$ is the temperature of the onset of the condensation, whereas this notation in textbooks is usually attributed to the end of the condensed phase.

One can see that the critical value $\kappa_{\mathrm{c}}=1.0$ separates two qualitatively different regimes. At $\kappa<\kappa_{\mathrm{c}}$, the curves $n(T)$ are continuous. While, at $\kappa>\kappa_{\mathrm{c}}$, they break down in two segments with a gap in between. This gap appears exactly between the densities $n_{1}$ and $n_{2}$, where $U(n)+m_{\pi}<0$, see Fig. 1 . For the parameter $\kappa=1.1$, the resulting solution for the particle density is depicted in Fig. 1 as a solid blue curve which consists of several segments. The lower branch at the critical temperature $T=T_{\mathrm{c}}$ is experiencing a jump from the value $n_{\mathrm{c}}=0.06 \mathrm{fm}^{-3}$ to the value $n_{2}=1.09 \mathrm{fm}^{-3}$. It is a consequence of the phase transition leading to the formation of the Bose condensate at this point. Because of the jump, this is certainly a first-order phase transition. It is rather obvious that, with a further increase of the temperature, the pion system will evolve along the horizontal line $n=n_{2}$ from $T_{\mathrm{c}}=115 \mathrm{MeV}$ up to $T_{2}=219 \mathrm{MeV}$, as shown in Fig. 1 (for $\kappa=1.1$ ). The critical temperature $T_{\mathrm{c}}$ is determined as a crossing point of the pressure curves for the liquid-gas and mixed phases. This is graphically shown in Fig. 2, which will be discussed in the next subsection. At $T>T_{\mathrm{c}}$, the branch of a self-consistent solution for the liquid-gas phase (blue dashed segment in Fig. 1) becomes metastable and exhibits a kind of the backhanding to the left, while approaching the limiting density $n_{\lim }(T)$ from below. Note that the upper segment (after the point marked as a "cross") is unstable.

If we reach the temperature $T_{\mathrm{c}}$ and continue to pump the energy into the multipion system, it will experience a phase transition leading to the formation of the Bose condensate, even in the system with $\mu=0$. As a consequence, the pion density will jump along the line $T=T_{\mathrm{c}}$.

Equation (12) is valid in our specific case where the evolution of the system goes along the constant density line $n=n_{2}$. Indeed, for every temperature $T$ from the interval $T_{\mathrm{c}}<T \leq T_{2}$ (see Fig. 1), the density of particles is $n=n_{2}$, and, thus, the value of the mean field is $U\left(n_{2}\right)=-m$. Due to this fact, we can rewrite Eq. (12) as

$n_{2}=n_{\text {cond }}(T)+n_{\lim }(T)$, 

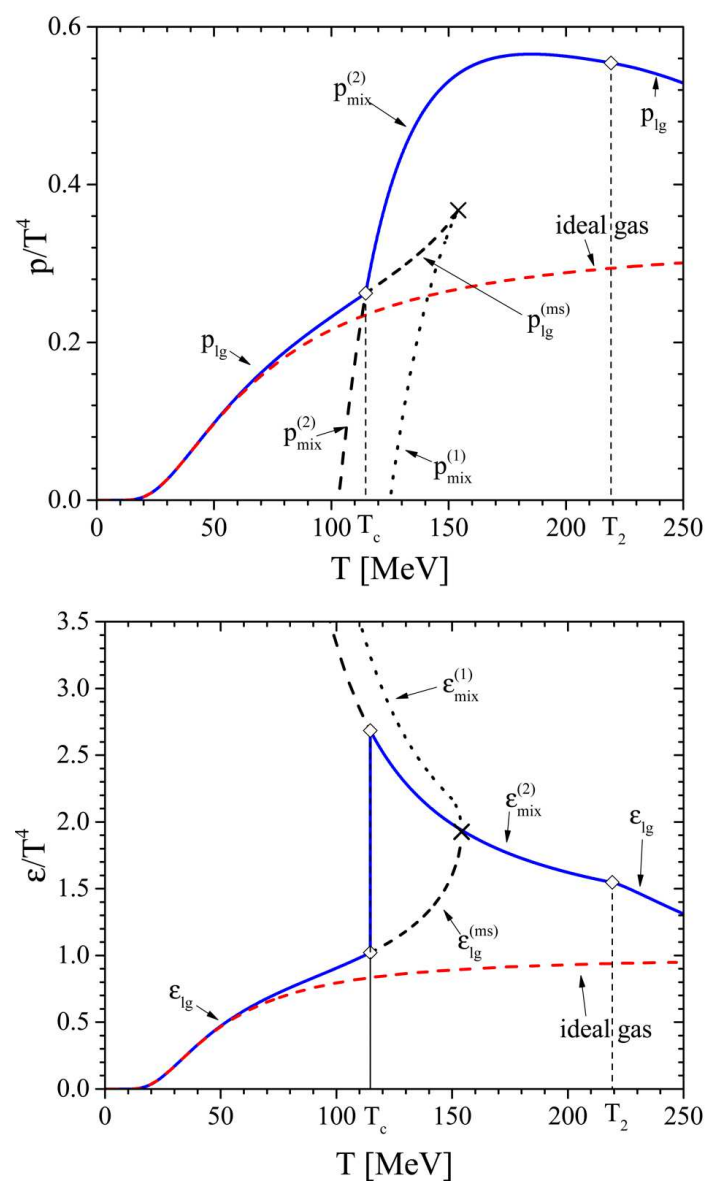

Fig. 2. Top panel: Pressure normalized to $T^{4}$ versus the temperature for an interacting pion system with $\mu=0$ and the attraction parameter $\kappa=1.1$. Solid blue lines labeled as $p_{\text {lg }}$ and $p_{\text {mix }}^{(2)}$ correspond to the pressure in the liquid-gas and mixed phases (along $n=n_{2}$ ), respectively, and represent the resulting equation of state. Lower panel: The energy density normalized to $T^{4}$ versus the temperature under the same conditions as in the left panel. The blue solid curve, which consists of several segments labeled as $\varepsilon_{\lg }$ and $\varepsilon_{\text {mix }}^{(2)}$ with a vertical segment along $T=T_{\mathrm{c}}$ represents the resulting equation of state. The energy density in the mixed phase $\varepsilon_{\text {mix }}^{(1)}$ calculated for the constant density $n=n_{1}$ is depicted as a dotted black line

where we use definition (7) of $n_{\lim }$. From Eq. (13), we see that the total particle density $n=n_{2}$ in the mixed phase consists, indeed, of the condensate contribution $n_{\text {cond }}$ and thermal particles $n_{\text {th }}$ which achieve the maximal particle density $n_{\lim }(T)$ at a given $T$. So, one should consider Eq. (12) as the self-consistent description of a pion condensate in the framework of the mean-field approach.

\subsection{Pressure and energy density in the mixed phase}

As we saw in the previous paragraph, the total particle density $n$ in the mixed phase with a Bose condensate is fixed at $n=n_{2}$. Thus, the pressure can be expressed as

$$
\begin{aligned}
& p_{\text {mix }}(T)=\frac{g}{3} \int \frac{d^{3} k}{(2 \pi)^{3}} \frac{\boldsymbol{k}^{2}}{\sqrt{m^{2}+\boldsymbol{k}^{2}}} \times \\
& \times\left\{\exp \left[\frac{\sqrt{m^{2}+\boldsymbol{k}^{2}}+U\left(n_{2}\right)}{T}\right]-1\right\}^{-1}+P_{\mathrm{ex}}\left(n_{2}\right),
\end{aligned}
$$

where $U\left(n_{2}\right)=-m$, and $P_{\mathrm{ex}}(n)$ is given by Eq. (9). One should bear in mind that the condensate particles with $k=0$ give no contribution to the kinetic part of the pressure (first term), but contribute to the interaction pressure via $P_{\mathrm{ex}}$ (second term). Figure 2 shows several branches of the pressure which represent different phases, and the resulting equation of state is depicted as a solid blue curve. The critical temperature, $T_{\mathrm{c}}=115 \mathrm{MeV}$, is obtained as the crossing point of two branches, representing the liquid-gas pressure for $\kappa=1.1$ and the pressure of the mixed phase at $n=n_{2}$. This explains why the Bose condensate appears only above $T_{\mathrm{c}}$, when the additional thermal pressure compensates the negative contribution of $P_{\mathrm{ex}}$. At $T>T_{\mathrm{c}}$, the liquid-gas branch $p_{\mathrm{lg}}^{(\mathrm{ms})}$ is metastable (dashed black curve in Fig. 2), the "cross" marks the turning-point. The dotted and dashed black lines labeled as $p_{\text {mix }}^{(1)}$ and $p_{\text {mix }}^{(2)}$ in Fig. 2 correspond to metastable states in the mixed phase for the pion densities $n=n_{1}$ and $n=n_{2}$, respectively.

In the mixed phase, the energy density consists of the kinetic part, $\varepsilon_{\text {kin }}(T)$, which is produced by thermal particles in the liquid-gas phase with density $n_{\lg }(T)$, and the condensate particles with density $n_{\text {cond }}(T)$, which have zero momentum. In accordance with the self-consistent solution of Eq. (12), the sum of these densities in the mixed phase remains constant, $n_{\lg }(T)+n_{\text {cond }}(T)=n_{2}$. This constant density $n_{2}$ determines the excess energy density $\varepsilon_{\text {ex }}\left(n_{2}\right)$ in the mixed phase. The specificity of the mixed phase is also in the fact that the mass of condensed particles produces another contribution to the energy density, $m n_{\text {cond }}(T)$. Using Eq. (4) with $U\left(n_{2}\right)=-m$ to obtain $\varepsilon_{\text {ex }}\left(n_{2}\right)=-m n-P_{\text {ex }}\left(n_{2}\right)$ and Eq. (13) to determine $n_{\text {cond }}(T)$, we can write the energy density in 
the mixed phase as

$$
\begin{aligned}
& \varepsilon_{\operatorname{mix}}^{(2)}=g \int_{|\boldsymbol{k}| \neq 0} \frac{d^{3} k}{(2 \pi)^{3}}\left(\sqrt{m^{2}+\boldsymbol{k}^{2}}-m\right) \times \\
& \times\left[\exp \left(\frac{\sqrt{m^{2}+\boldsymbol{k}^{2}}-m}{T}\right)-1\right]^{-1}-P_{\mathrm{ex}}\left(n_{2}\right) .
\end{aligned}
$$

In Fig. 2 (lower panel), we present the energy densities normalized to $T^{4}$ at $\kappa=1.1$ in different phases: the resulting equation of state of the interacting pions in the temperature interval $0<T<250 \mathrm{MeV}$ (blue solid curve which includes the jump due to the phase transition at $T_{\mathrm{c}}$ ); in the mixed phase, the energy density of pions in metastable state $\varepsilon_{\mathrm{mix}}^{(1)}$ calculated for the constant density $n=n_{1}$ (black dotted curve); the energy density of meta-stable states in the liquid-gas phase $\varepsilon_{\mathrm{lg}}^{(\mathrm{ms})}$ (blue dashed segment); and the energy density of the ideal pion gas at $\mu=0$ (red dashed curve). One can see that, for $\kappa=1.1$, the model predicts the upward jump of the energy density of about $30 \mathrm{MeV} / \mathrm{fm}^{3}$ (latent heat) at the critical temperature $T_{\mathrm{c}}=115 \mathrm{MeV}$. This is another manifestation of the first-order phase transition.

\section{Concluding Remarks}

In this paper, we have presented a thermodynamically consistent method to describe dense bosonic systems at high temperatures and the zero chemical potential. A central step of this approach is to solve the self-consistent equation (6) for the pion density at a given temperature in the presence of the meanfield $U(n)$ given by Eq. (8). Note that the integral in Eq. (6) is positive definite, only if the condition $U(n) \geq-m$ is fulfilled. If the attractive mean field is so strong that this condition is violated, the multiboson system develops a Bose condensate. Our analysis leads to the conclusion that, in the presence of a condensate, the allowed states of the system must satisfy the condition $U(n)+m=0$, where $n$ is the total particle density including the condensate. This very unusual behavior is possible, only if the attractive interaction between bosons is strong enough. However, the empirical data and theoretical calculations show that the pion-pion interaction is rather weak at energies $\leq 100 \mathrm{MeV}$. Nevertheless, an additional contribution to the pion mean field can be provided by the attractive pion-nucleon interaction in cold nuclear matter, as demonstrated in Refs. [1-4], or by $\rho$-mesons and baryon-antibaryon pairs at high temperatures, as considered in Refs. [25, 26]. It is obvious that many other hadronic species will be present in the hadronic matter at high temperatures. They may induce additional attractive contributions to the mean field felt by pions. This will add new terms to the pion optical potential, which are proportional to the density of these species, see, e.g., Ref. [25]. These terms will reduce the pion effective mass and, thus, the threshold for the appearance of a pion condensate, as given by Eq. (10). Another interesting possibility studied in Refs. [8-13] is the Bose condensation in a pure pionic system with non-zero isospin chemical potential. We are planning to consider such interacting systems in the future.

Finally, we would like to point out that, at first glance, it seems that the mesonic degrees of freedom may not be appropriate at high particle densities $\left(n \approx 1 \mathrm{fm}^{-3}\right)$ and high temperatures $(T \geq 150$ $\mathrm{MeV})$, as considered in this paper. Meanwhile, the temperature of condensation, $T_{\mathrm{c}} \approx 115 \mathrm{MeV}$, lies in a well-determined hadronic sector. Hence, the melting of pions into quarks and antiquarks during the heating of the system can go directly from the dense phase of condensed pions. Vice versa, during the cooling of the system, soft pions can form a condensate on the stage of hadronization.

The work of D.A. and D.Z. was supported by the Program "The structure and dynamics of statistical and quantum field systems" of the Department of Physics and Astronomy of the NAS of Ukraine. I.M. acknowledges the financial support from Helmholtz International Center for FAIR, Germany. The work of O.S. has been supported by the scientific program "Astronomy and space physics" (project 19БФ023-01) of Taras Shevchenko National University of Kyiv. H.S. appreciates the support from J.M. Eisenberg laureatus chair.

1. A.B. Migdal. Stability of vacuum and limiting fields. Sov. Phys. JETP 34 (6), 1184 (1972).

2. A.B. Migdal, O.A. Markin, I.N. Mishustin. The pion spectrum in nuclear matter and pion condensation. Sov. Phys. JETP 39 (2), 212 (1974).

3. A.B. Migdal. Pion fields in nuclear matter. Rev. Mod. Phys. 50, 107 (1978).

4. A.B. Migdal, E. Saperstein, M. Troitsky, D. Voskresensky. Pion degrees of freedom in nuclear matter. Phys. Rept. 192, 179 (1990). 
5. A. Anselm, M. Ryskin. Production of classical pion field in heavy ion high energy collisions. Phys. Lett B 226, 482 (1991).

6. J.-P. Blaizot, A. Krzywicki. Soft-pion emission in highenergy heavy-ion collisions. Phys. Rev. D 46, 246 (1992).

7. J.D. Bjorken. A full-acceptance detector for SSC physics at low and intermediate mass scales: an expression of interest to the SSC. Intern. J. Mod. Phys. A 7, 4189 (1992).

8. I.N. Mishustin, W. Greiner. Multipion droplets. J. Phys. G: Nucl. Part. Phys. 19, L101 (1993).

9. D.T. Son, M.A. Stephanov. QCD at finite isospin density. Phys. Rev. Lett. 86, 592 (2001).

10. J. Kogut, D. Toublan. QCD at small non-zero quark chemical potentials. Phys. Rev. D 64, 034007 (2001); [arXiv:hepph/0103271].

11. D. Toublan, J. Kogut. Isospin chemical potential and the QCD phase diagram at nonzero temperature and baryon chemical potential. Phys. Lett. B 564, 212 (2001); [arXiv:hep-ph/0301183].

12. A. Mammarella, M. Mammarelli. Intriguing aspects of meson condensation. Phys. Rev. D 92, 085025 (2015); [1507.02934 [hep-ph]], 2015.

13. S. Carignano, L. Lepori, A. Mammarella, M. Mannarelli, G. Pagliaroli. Scrutinizing the pion condensed phase. Eur. Phys. J. A 53, 35 (2017); [arXiv:1610.06097 [hep-ph]].

14. B.B. Brandt, G. Endrodi. QCD phase diagram with isospin chemical potential. PoS LATTICE2016 039 (2016); [arXiv:1611.06758 [hep-lat]].

15. B.B. Brandt, G. Endrodi, S. Schmalzbauer. QCD at finite isospin chemical potential. arXiv:1709.10487 [hep-lat].

16. L.M. Satarov, M.N. Dmitriev, I.N. Mishustin. Equation of state of hadron resonance gas and the phase diagram of strongly interacting matter. Phys. Atom. Nucl. 72, 1390 (2009).

17. Volodymyr Vovchenko, Anton Motornenko, Paolo Alba, Mark I. Gorenstein, Leonid M. Satarov, Horst Stoecker. Multicomponent van der Waals equation of state: Applications in nuclear and hadronic physics. Phys. Rev. C 96, 045202 (2017).

18. D. Anchishkin, V. Vovchenko. Mean-field approach in the multi-component gas of interacting particles applied to relativistic heavy-ion collisions. J. Phys. G: Nucl. Part. Phys. 42, 105102: 1-27 (2015); arXiv:1411.1444 [nucl-th].

19. D.V. Anchishkin. Particle finite-size effects as a mean-field approximation. Sov. Phys. JETP 75, 195 (1992).
20. D. Anchishkin, E. Suhonen. Generalization of meanfield models to account for effects of excluded-volume. Nucl. Phys. A 586, 734 (1995).

21. R.V. Poberezhnyuk, V. Yu. Vovchenko, D.V. Anchishkin, M.I. Gorenstein. Limiting temperature of pion gas with the van der Waals equation of state. J. Phys. G: Nucl. Part. Phys. 43, 095105 (2016); arXiv:1508.04585 [nucl-th].

22. D. Anchishkin, I. Mishustin. H. Stoecker. Phase transition in interacting boson system at finite temperatures. J. Phys. G 46, 035002 (2019); arXiv:1806.10857 [nucl-th].

23. J.P. Hansen, I.R. McDonald. Virial expansion of the equation of state. In Theory of Simple Liquids (Academic Press, 2006), p. 75, Eq. (3.9.14).

24. A.J. Leggett. Quantum Liquids (Oxford University Press Inc., 2006).

25. E.V. Shuryak. Collective interaction of mesons in hot hadronic matter. Nucl. Phys. A 533, 761 (1991).

26. J. Theis, G. Graebner, G. Buchwald, J. Maruhn, W. Greiner, H. Stoecker, J. Polonyi. Phase transition of the nucleon-antinucleon plasma in a relativistic mean-field theory. Phys. Rev. D 28, 2286 (1983).

Received 22.10.19

Д. Анчишкін, I. Мішустін,

О. Сташко, Д. Журавель, Х. Штокер

БОЗЕ-ЕЙНШТЕЙНІВСЬКА

КОНДЕНСАЦЯ У СИСТЕМІ ВЗАЄМОДІЮЧИХ

БОЗОНІВ ПРИ СКІНЧЕННИХ ТЕМПЕРАТУРАХ

$\mathrm{P}$ е $з$ ю м е

Термодинамічні властивості системи взаємодіючих бозонів при скінченних температурах та нульовому хімічному потенціалі є об'єктом дослідження в рамках наближення середнього поля, яке моделюється у вигляді потенціала Скірма. Вважається, що середнє поле містить як притягуючу компоненту, так і відштовхуючу. Отримано самоузгоджені співвідношення між середнім полем та термодинамічними функціями. Показано, що коли притягання є досить сильним, то система має фазовий перехід першого роду, при якому виникає бозе-конденсат. Конденсована фаза характеризується постійною загальною густиною частинок. Показано, що при критичній температурі густина енергї має стрибок. 